\title{
Relaciones familiares, calidad de vida y competencia social en adolescentes y jóvenes colombianos
}

\section{Family relationships, quality of life and social competence in Colombian adolescents and young adults}

\author{
Sonia Carrilloํㅗ Karen Ripoll-Nuñez, Victoria Cabrera y Hammer Bastidas \\ Universidad de Los Andes. Bogotá - Colombia
}

(Recepción: Octubre 2009 - Aceptación: Diciembre 2009)

\begin{abstract}
Resumen
El propósito principal del estudio fue evaluar el papel de las relaciones afectivas dentro de la familia y su influencia en dos aspectos centrales del funcionamiento del individuo: la competencia social y la calidad de vida, en una muestra de 103 adolescentes y jóvenes adultos colombianos. Los resultados mostraron asociaciones significativas diferentes para hombres y mujeres entre los subsistemas familiares, la calidad de vida y la competencia social de los participantes. Se discuten los resultados con base en la literatura sobre efectos diferenciales de las relaciones familiares en el bienestar psicológico y social de los adolescentes y jóvenes.

Palabras Claves: Relaciones familiares, calidad de vida y competencia social
\end{abstract}

\begin{abstract}
The main purpose of this study was to assess the influence of family relationships on two central aspects of individual functioning: social competence and quality of life in a sample of 103 Colombian adolescents and young adults. Results revealed different significant associations for men and women between the study variables (relationships with parents and sibling, quality of life and social competence). Results are discussed based on the literature on differential effects of family relationships on adolescents' and young adults' psychological and social well-being.

Key words: Family relationships, social competence and quality of life.
\end{abstract}

1 Correspondencia: Sonia Carrillo. Departamento de Psicología, Universidad de los Andes. Dirección Cra 1 E \# 18 A- 10 , Bogotá Colombia. Teléfono: 5713324365 Email: scarrill@uniandes.edu.co 
La adolescencia, se ha concebido tradicionalmente como una de las etapas más difíciles en el desarrollo del individuo. Durante mucho tiempo se identificó a la adolescencia como una "etapa de crisis" en lugar de una etapa de transición o de transformaciones particulares. Esta concepción negativa llevó a muchos investigadores a dar un lugar central en los estudios sobre adolescencia a características negativas (rebeldía, problemas de comportamiento, dificultades en las relaciones con padres, etc.) y las características positivas asociadas a los cambios físicos, cognoscitivos o sociales estuvieron relegadas. En las últimas décadas esta concepción ha cambiado, así como los problemas de investigación e igualmente se ha ampliado el espectro de los estudios tanto en la adolescencia como en la adultez joven.

Una de las áreas que ha retomado una importancia creciente en los estudios sobre adolescentes es el área del desarrollo social y afectivo. Dentro de este tema de investigación se han explorado las características de las relaciones afectivas dentro del contexto familiar (con padres y hermanos) y su influencia en diferentes aspecto del funcionamiento de los adolescentes. Por ejemplo se han evaluado en detalle las conexiones entre las características de las relaciones con los padres y el desempeño social de niños y jóvenes. Aspectos asociados a las relaciones entre hermanos han sido menos investigados.

Recientemente se ha observado un interés en la literatura psicológica en general, por el estudio de indicadores y factores asociados al bienestar y a la calidad de vida de los individuos. Este interés se ha transmitido igualmente a los investigadores del desarrollo adolescente, lo cual refleja un cambio en la tendencia de los problemas investigados y un mayor énfasis en analizar aspectos positivos más que negativos en esta etapa en particular. Aunque la literatura en este sentido es aún limitada, se encuentran modelos explicativos y contribuciones importantes y alrededor del estudio de la calidad de vida en estas etapas del desarrollo.

La presente investigación surge en consonancia con el reciente interés por explorar dimensiones positivas del desarrollo social adolescente y por profundizar en la comprensión de aquellos factores que inciden en el funcionamiento social de los jóvenes, así como en la valoración de su bienestar. Es así como el propósito principal del estudio se centró en la evaluación del papel de las relaciones afectivas dentro de la familia y su influencia en dos aspectos centrales del funcionamiento del individuo: la competencia social y la calidad de vida, en una muestra de adolescentes y jóvenes adultos colombianos.

\section{Relaciones padres-hijos y calidad de vida}

La calidad de vida es un constructo multidimensional que ha sido desarrollado para dar cuenta de la percepción de bienestar y del grado de satisfacción de las personas en distintos ámbitos de su funcionamiento (Diener, Suh, Lucas \& Smith, 1999). Sin embargo, este constructo ha sido utilizado con frecuencia para referirse solamente a aspectos relacionados con el ámbito de la salud o como un mero complemento de conceptos más tradicionales tales como funcionamiento general y estándar de vida (WHOQOL, 1998; Skevington, 2002; Power, 2003).

La literatura médica ha producido una cantidad significativa de investigaciones sobre calidad de vida relacionada con la salud, con el propósito principal de identificar las repercusiones que las enfermedades tienen en la vida de las personas. Las propuestas conceptuales más recientes sobre calidad de vida presentan una perspectiva más amplia y se centran en la identificación de diferentes factores (tanto del individuo como del contexto) relacionados con el bienestar físico y emocional, las relaciones interpersonales, el funcionamiento social, los recursos materiales y económicos, y el contexto geográfico y social (Power, et al., 1999; Diwan, 2000; Power, 2003; Botello-Harbaum, et al., 2008; Gringeri \& Von Mackensen, 2008). Dentro de esta revisión del concepto de calidad de vida, otros teóricos han enfatizado su relación con otros conceptos tales como felicidad y la satisfacción con la vida (Zimmermann, Eisemann \& Fleck, 2007). 
La literatura sobre el desarrollo adolescente se ha centrado en la evaluación de diferentes características individuales, y en factores del ambiente social que influyen en el funcionamiento y en el ajuste psicológico de los jóvenes. El ambiente social se ha definido como un sistema complejo de relaciones con diferentes personas las cuales se establecen dentro de subsistemas, como el familiar, el escolar y el de los pares (Parke, 2004). Dentro del ambiente social se ha dado especial énfasis al estudio de dimensiones particulares de las relaciones con los padres y a la valoración de las vías de influencia entre dichas dimensiones y el ajuste psicológico, la competencia social y la adaptación académica de los adolescentes. Una menor atención se ha dedicado a otros subsistemas como el de las relaciones entre hermanos y al papel que dichas relaciones juegan en el desarrollo de los jóvenes.

La importancia de las relaciones familiares en el proceso de socialización y adaptación de los niños y los adolescentes ha sido ampliamente documentada. Existe un cuerpo amplio de literatura que provee evidencia de asociaciones significativas entre características positivas de la interacción entre padres e hijos (i.e. altos niveles de aceptación y de apoyo percibidos, patrones de crianza positivos y estilos de apego seguros) y altos niveles de ajuste psicológico y de competencia social y alta calidad en las relaciones con pares y amigos en niños y adolescentes. Dicha literatura ha enfatizado igualmente la influencia de patrones inadecuados de relación entre padres e hijos en el desarrollo de problemas de comportamiento y desadaptación en los hijos (Musitu \& Cava, 2002; Rohner, 2005; Ladd, 2005; Sroufe, Egeland, Carlson \& Collins, 2005; Moss, St-Laurent, Dubois \& Cyr, 2005; Laursen \& Mooney, 2008; Berlin, Cassidy \& Appleyard, 2008).

Durante la última década se ha observado un giro interesante en el foco de las investigaciones alrededor de la adolescencia hacia diferentes aspectos del funcionamiento no contemplados hasta el momento; uno de estos aspectos es el estudio del bienestar y la calidad de vida. Los estudios muestran un interés creciente de los investigadores por ampliar la comprensión tanto teórica como empírica de los factores asociados a la calidad de vida de los adolescentes y de los jóvenes adultos (Rice \& Cummings, 1996; Edwards \& LópezVera, Thakral, González, \& cols, 2008 ); dentro de este interés, la exploración del papel que juegan las relaciones familiares ha sido crucial. Sin embargo, los cambios en las concepciones de familia, la complejidad de las dinámicas familiares y la diversidad en el tipo y calidad de las relaciones entre los miembros de un grupo familiar han llevado a que el estudio de su influencia en la calidad de vida de los hijos se haya abordado desde diferentes ángulos y con diferentes énfasis (Orbuch, et al., 2005; Zimmermann, Eisemann \& Fleck, 2007). Con respecto a las relaciones padres-hijos, algunos autores se han centrado en el estudio de los estilos parentales y han encontrado que los adolescentes que son criados en contextos familiares que promueven un estilo parental autoritativo reportan mayores niveles de felicidad y mayor satisfacción con su calidad de vida (Furnham \& Cheng, 2000; Cheng \& Furnham, 2004; Zimmermann, Eisemann \& Fleck, 2007; Botello-Harbaum, et al., 2008).

Si bien la mayoría de los estudios ha analizado la influencia de las relaciones padres-hijos como un todo, algunos autores han explorado de manera más detallada el efecto diferencial de la interacción con cada uno de los padres en la calidad de vida de los adolescentes. Por ejemplo, Furnham \& Cheng (2000) evaluaron la influencia del estilo parental de la madre y del padre en la valoración del nivel de felicidad en una muestra de 406 adolescentes y adultos jóvenes. Estos autores encontraron que sólo el estilo autoritativo de la madre predecía directamente el grado de felicidad reportado por los participantes. Pero, al excluir el estilo parental de la madre, se encontró que el estilo autoritativo del padre adquiría un poder predictor directo del grado de felicidad de los hijos. El análisis de otras dimensiones de la conducta parental de padres y madres muestra que el exceso de control por parte del padre tiene una influencia negativa y significativa sobre la felicidad de los adolescentes, pero esta influencia se encuentra mediada por la auto-evaluación positiva que los hijos hacen de sí mismos, mientras que el grado de cuidado materno influye significativamente en la felicidad reportada por los hijos, y, este efecto está mediado por el auto-criticismo y la auto-evaluación positiva (Cheng \& Furnham , 2004) 
Otros estudios con poblaciones clínicas (adolescentes y adultos jóvenes con cáncer) han añadido evidencia que apoya la influencia diferencial de características de la interacción con cada uno de los padres en el bienestar de los jóvenes. Estos estudios han mostrado que, aunque la calidad de la relación con el padre no fue reportada por los participantes de forma tan positiva como la relación con la madre, sí se encontró una mayor influencia de la relación padre-hijo en diversos aspectos de la calidad de vida (calidad de vida global, bienestar psicológico y bienestar espiritual), mientras que, la calidad de la relación con la madre, a pesar de ser mejor evaluada, sólo ejercía un efecto significativo sobre el bienestar psicológico (Orbuch, et al., 2005). Los hallazgos de Zimmermann, Eisemann \& Fleck (2008) contradicen en parte el anterior resultado; estos autores encontraron que la calidez emocional de la madre se asoció significativamente con la calidad de vida global, el bienestar psicológico y la satisfacción con las relaciones sociales, mientras que la percepción de rechazo, fue el único aspecto de la interacción con el padre que mostró un efecto negativo en el bienestar psicológico de los hijos.

La influencia de las relaciones familiares en el bienestar y la calidad de vida en etapas posteriores a la niñez se ha abordado desde otras perspectivas teóricas. Desde finales de los años 90 un grupo de teóricos del apego inició una exploración sistemática de los nexos entre el apego y la calidad de vida en la etapa de la adolescencia tardía; dichos investigadores llevaron a cabo diferentes estudios en los que evaluaban las posibles relaciones y modos de influencia de las características de las relaciones de apego entre padres e hijos y la valoración que los hijos tienen de su bienestar social y su calidad de vida. Hallazgos de algunos de estos estudios indicaron conexiones significativas entre la percepción que adolescentes y jóvenes tienen de las relaciones de apego que han establecido con sus padres y diferentes dimensiones de su bienestar emocional, psicológico y social (Armsden \& Geenberg, 1987; Kenny \& Rice, 1995; Rice\& Cummins, 1996).

\section{Calidad de la relación entre hermanos y competencia social}

La literatura sobre familia y sobre el impacto que las relaciones familiares tienen en el desarrollo de los hijos se ha centrado fundamentalmente en el sistema parental. Durante muchos años dominó la tendencia a considerar solamente a los padres y a las relaciones de los padres con los hijos como las únicas fuentes de influencia en las diferentes áreas de funcionamiento de niños y adolescentes. Actualmente, existe un mayor interés investigativo por: a)incluir otros subsistemas familiares en los estudios (relación de pareja, relación entre hermanos, relación con familia extensa), b) por entender las relaciones que se generan entre los distintos miembros y los subsistemas que componen la familia, c) por explicar el impacto o influencia que dichas relaciones tienen sobre el ajuste y el bienestar de los adolescentes (Oliva \& Arranz, 2005; Noller, 2005).

Algunos autores consideran que las relaciones entre hermanos revisten una importancia significativa dentro del estudio de las relaciones afectivas cercanas y del desarrollo social y emocional. Diversos argumentos se han emitido para sustentar dicha afirmación. Por ejemplo, las relaciones entre hermanos son las relaciones más largas que tiene un individuo (Noller, 2005); los hermanos pueden cumplir el papel protector y de cuidado de las figuras de apego (Thrinke \& Bartholomew, 1997; Davis, 2002;); las relaciones entre hermanos se constituyen en un contexto de aprendizaje de habilidades sociales (Dunn, 1994); las relaciones entre hermanos pueden actuar como un factor protector contra efectos de situaciones familiares adversas (Brody, 2004; Lokwood y cols., 2002), entre otros.

Los estudios sobre relaciones entre hermanos se han centrado en dos problemas principales. Primero, en la identificación de las características y dimensiones que definen el tipo y la calidad de las relaciones entre los hermanos/as. Con respecto a este punto, Buhrmester \& Furman (1990) definieron a las relaciones entre hermanos como caracterizadas por factores como calidez, conflicto, definición de estatus y rivalidad. Con base en estas dimensiones diseñaron un instrumento para evaluar la calidad de la relación entre hermanos (Cuestionario de Relaciones entre Hermanos- SRQ por su sigla en inglés). Un segundo tema de estudio ha sido la evaluación de la influencia de la calidad de la relación entre hermanos/as en el desarrollo y la competencia social de los niños y los 
adolescentes. En este sentido se ha encontrado que los adolescentes que reportan altos niveles de comunicación y reciprocidad en la relación con sus hermanos/as, presentan mayores habilidades para iniciar interacciones con otros y exhiben mayores niveles de empatía en sus relaciones con otros (Dunn , 2002; Lockwood, et al., 2006).

Una pregunta interesante que ha guiado a algunos investigadores se ha centrado en la evaluación de las características diferenciales de las relaciones de acuerdo con el sexo de los hermanos. McCoy, et al., (2002), por ejemplo, encontró que la relación entre hermanas adolescentes está caracterizada por altos niveles de calidez, confianza y reciprocidad, mientras que las relaciones entre hermanos presentan mayores niveles de conflicto.

Estudios realizados en diadas o grupos de hermanos permiten establecer que una relación filial funcional suministra a los niños un conjunto de beneficios tales como seguridad, apoyo, regulación emocional, compañía, cooperación, entre otros. Asimismo, este tipo de relaciones promueven un desarrollo de expectativas igualmente positivas frente a las interacciones con los demás (Lamarche, et al., 2006) y, al mismo tiempo, influyen en el ajuste y adaptación tanto a nivel personal como social de los adolescentes (Oliva \& Arranz, 2005). Igualmente, también se ha observado una influencia de los patrones de interacción y de conducta negativa de un hermano en el desempeño social y el ajuste del otro (Lamarche, et al., 2006).

Finalmente, se ha observado que durante la adolescencia las relaciones positivas con hermanos también contribuyen al buen funcionamiento de las relaciones con padres y pares. Todo esto genera, a su vez, un incremento de autoestima y satisfacción de vida, especialmente en las mujeres (Oliva \& Arranz, 2005). Estos resultados son explicados al considerar que las mujeres tienen una mayor sensibilidad hacia las relaciones interpersonales, principalmente dentro de los ambientes familiares,

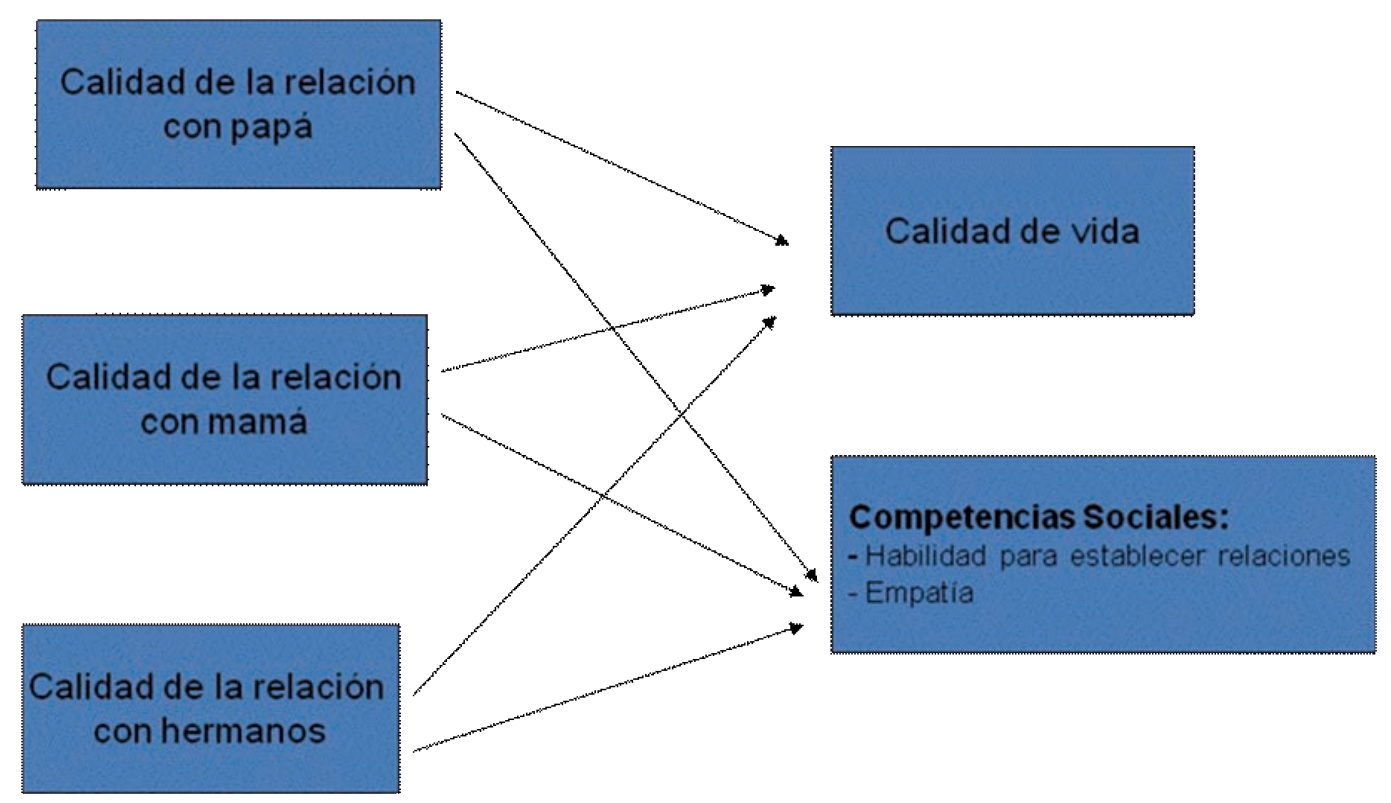

Figura 1. Diseño del estudio. 
a diferencia de los hombres adolescentes quienes se preocupan más por su rendimiento en el campo académico o deportivo (Oliva \& Arranz, 2005).

El propósito central de este estudio fue evaluar la influencia de la calidad de la relación con padres y hermanos en la calidad de vida y la competencia social de una muestra de adolescentes y jóvenes adultos colombianos residentes en Bogotá. El diseño del estudio aparece en la Figura 1.

En este estudio se examinaron las siguientes preguntas de investigación:

1. Cuál es la asociación que existe entre la percepción de la calidad de la relación con los padres y con los hermanos y la calidad de vida de los participantes?

2. Cuál es la asociación que existe entre la percepción de la calidad de la relación con los padres y con los hermanos y la competencia social de los participantes?

3. Cuál es el efecto de la percepción de la calidad de la relación con los padres y con los hermanos en la calidad de vida de los adolescentes y jóvenes adultos?

4. Cuál es el efecto de la percepción de la calidad de la relación con los padres y con los hermanos en las habilidades para establecer relaciones y en el nivel de empatía de los adolescentes y jóvenes adultos?

5. Existen diferencias en las variables del estudio dependiendo del sexo de los participantes y del sexo del hermano seleccionado?

Las hipótesis que se evaluaron con base en estas preguntas fueron las siguientes:

Hipótesis 1: Existe una relación estadísticamente significativa entre la percepción positiva de la relación con los padres y con los hermanos y el nivel de calidad de vida de los participantes.

Hipótesis 2: Existe una relación estadísticamente significativa entre la percepción positiva de la relación con los padres y con los hermanos y las habilidades sociales de los participantes (habilidad para establecer relaciones y empatía).

Hipótesis 3: Existe un efecto estadísticamente significativo y positivo de la calidad de la relación con cada uno de los padres y el/la hermano/a sobre el nivel de calidad de vida de los participantes.

Hipótesis 4: Existe un efecto estadísticamente significativo y positivo de la percepción de la relación con cada uno de los padres y con el/la hermano/a sobre las habilidades sociales de los participantes (habilidad para establecer relaciones y empatía).

\section{Método}

\section{Participantes}

La muestra estuvo conformada por 103 adolescentes y jóvenes adultos (41 hombres y 62 mujeres) cuyas edades oscilaron entre 15 y 25 años $(M=19,3, D E=2,14)$; todos los participantes pertenecen a familias nucleares o tradicionales y residen en la ciudad de Bogotá. La totalidad de los participantes eran estudiantes (de educación secundaria o de universidad) y solamente $2 \%$ de ellos, reportaron tener un trabajo adicional a su actividad universitaria. Información adicional sobre variables sociodemográficas de los participantes del estudio se encuentra en la Tabla 1. 
Tabla 1 - Estadísticos descriptivos y Prueba t para muestras independientes

\begin{tabular}{lccc}
\hline Variables del estudio & $\begin{array}{c}\text { Hombres } \\
(\mathrm{N}=41) \\
M\end{array}$ & $\begin{array}{c}\text { Mujeres } \\
(\mathrm{N}=62) \\
M\end{array}$ & $g l=101$ \\
\hline Calidad relación papá & $\begin{array}{c}76,15 \\
(12,05)\end{array}$ & $\begin{array}{c}72,37 \\
(11,34)\end{array}$ & 1,59 \\
Calidad relación mamá & 79,85 & 80,95 &,- 47 \\
& $(10,70)$ & $(11,55)$ & $-1,56$ \\
Calidad relación hermano & 3,52 & 3,74 &,- 17 \\
Calidad de vida & $(, 69)$ & $(, 72)$ & \\
Habilidad para establecer relaciones & 3,91 & 3,92 &,- 08 \\
& $(, 39)$ & $(, 48)$ & $-1,34$ \\
Empatía & 3,13 & 3,15 & $(, 85)$ \\
\hline
\end{tabular}

Nota. Los valores en paréntesis corresponden a las desviaciones estándar.

\section{Variables e Instrumentos}

Variables sociodemográficas: Se diseñó un cuestionario breve para recoger información sociodemográfica de los participantes como la edad, sexo, nivel educativo, estrato socioeconómico, así como algunos datos de sus familias (edad y nivel educativo de los padres, edad de los hermanos, etc.)

Calidad de la Relación con los Padres: Para evaluar la percepción de los participantes sobre la calidad de la relación con los padres se utilizó el Inventario de Apego para Padres y Pares (Inventory of Parent and Peer Attachment - IPPA- de Armsden \& Greenberg, 1987). Este instrumento incluye 3 versiones que evalúan la percepción de la relación con la madre, con el padre y con los pares respectivamente. En el presente estudio se utilizaron las dos primeras versiones solamente. Cada versión del IPPA consta de 25 items que se califican con una escala Likert de 5 puntos, donde $1=$ Nunca es cierto y 5 = Casi siempre es cierto. Los items evalúan dos dimensiones positivas de la relación (confianza y comunicación) ("My mamá respeta mis sentimientos"; "Yo le cuento a mi mamá sobre mis problemas y preocupaciones”)y una dimensión negativa de la relación (alienación) (“ Yo me molesto fácilmente cuando estoy cerca de mi mamá"); puntajes altos en cada una de estas dimensiones indican niveles altos de esa característica en la relación con cada uno de los padres. Para efectos de los análisis del presente estudio se utilizaron solamente las dos dimensiones positivas del IPPA. La dimensión de confianza mostró índices adecuados de consistencia interna $\left(_{-}=, 77\right.$ para la relación con mamá y _ = ,87 para la relación con papá); los índices de consistencia interna para la dimensión de comunicación fueron igualmente adecuados $\left(_{-}=, 85\right.$ para la relación con mamá y _ = ,87 para la relación con papá). Basados en estas dos dimensiones se creó un puntaje total de la calidad de la relación con cada uno de los padres, el cual se obtuvo de la suma de los puntajes dados por los participantes a los ítems correspondientes a confianza y a comunicación. 
Calidad de la Relación con los Hermanos. Para evaluar la percepción de los participantes sobre la calidad de la relación con los hermanos se utilizó el Cuestionario de Relación entre Hermanos (Sibling Relationship Questionnaire- SRQ Furman \& Buhrmester, 1985). El cuestionario original consta de 48 ítems que evalúan 3 dimensiones principales: calidez/cercanía ("¿Qué tanto tu hermano(a) y tú se lo cuentan todo?”), poder relativo/estatus (“¿Qué tanto tu hermano te dice que hacer?”), y conflicto/rivalidad (“¿Qué tanto tu hermano y tú entran en desacuerdo y discuten entre sí?”). Los ítems se califican utilizando una escala tipo Likert de 5 puntos, donde $1=$ casi nunca y 5 = mucho. En el presente estudio se incluyeron 8 ítems de la dimensión de calidez; éstos ítems mostraron una consistencia interna adecuada $\left({ }_{-}=, 87\right)$.

Competencia Social. Se empleó el Interpersonal Competence Questionnaire (ICQ) (Buhrmester, Furman, Wittenberg \& Reis, 1988). Este instrumento consta de 40 items que se agrupan en cinco dimensiones: (a) Habilidad para establecer relaciones (“¿Qué tan bueno(a) eres para sugerir a alguien a quien acabas de conocer hacer algo juntos, como por ejemplo ir a un partido o a cine?”) ,(b) Revelar información personal ("¿Qué tan bueno(a) eres para contarle a otras personas cosas que te avergüenzan acerca de ti mismo(a)?”), (c) Comunicación Asertiva (“¿Qué tan bueno(a) eres a la hora de defenderte ti mismo(a)?”), d) Empatía (“¿Qué tan bueno(a) eres para demostrar que realmente te interesan los problemas de los demás?”), y (e) Manejo de Conflictos Interpersonales (“ ¿Qué tan bueno(a) eres manejando los desacuerdos, de forma que al final una persona no siempre salga perdiendo?"). En este estudio se emplearon 16 ítems que corresponden a las dimensiones Habilidad para establecer relaciones y Empatía. Los ítems se califican con una escala tipo Likert de 5 puntos, donde $1=$ =deficiente y $5=$ extremadamente bueno. Los índices de consistencia interna de las dos dimensiones fueron adecuados (_ =,90 y ,87 respectivamente).

Calidad de Vida: Esta variable fue evaluada a través de Escala de Bienestar Subjetivo (Vahos, 1995). Este instrumento consta de 63 items que evalúan el grado de satisfacción que siente la persona en diferentes ámbitos de su vida. Los ítems se califican con una escala Likert de 6 puntos en donde 1= No aplica hasta 6= Muy satisfecho. En el presente estudio se excluyeron 7 items de la versión original. Para efectos de los análisis se seleccionaron solamente 3 dimensiones particulares (calidad de vida familiar, social y de pareja), ("Qué tan satisfecho está usted con respecto a: a) las expectativas que mi familia tiene respecto a mí; las relaciones con mis compañeros; el afecto que me ofrece mi pareja”); estas dimensiones mostraron buenos índices de consistencia interna (_ = ,89, ${ }_{-}=, 83, y_{-}=, 87$ respectivamente). Se creó un puntaje total de calidad de vida que correspondió a la suma de los puntajes dados por los participantes a los ítems de las 3 dimensiones mencionadas.

\section{Procedimiento}

Los participantes del estudio fueron contactados a través de dos procedimientos principales: 1) Visitas a diferentes cursos en dos universidades de la ciudad; 2) Bola de nieve. A los jóvenes interesados en participar en el estudio se les entregó una carta en la que se explicaba el propósito del estudio, las características de su participación y el carácter confidencial y anónimo de la información recolectada. Una vez obtenidos los consentimientos de los participantes y sus padres los adolescentes y jóvenes contestaron un cuestionario que contenía los ítems de los diferentes instrumentos incluidos en el estudio. Para responder a las preguntas que evaluaron la calidad de la relación entre hermanos los participantes debían seleccionar uno de sus hermanos y evaluar los diferentes aspectos de la relación teniendo en mente ese hermano en particular. La duración de su participación fue de 45 minutos.

\section{Resultados}

El presente estudio tuvo como propósito central evaluar la percepción del nivel de calidad de vida y de competencia social de adolescentes y jóvenes adultos pertenecientes a familias tradicionales o nucleares y evaluar el papel de la calidad de la relación con padres y hermanos en dichas variables. Los resultados de este estudio se presentarán siguiendo las principales hipótesis planteadas alrededor de las variables investigadas. En primer lugar se presentarán los estadísticos descriptivos 
de las variables estudiadas; en segundo lugar se presentarán los resultados obtenidos en los análisis que evaluaron las diferentes relaciones entre las mismas.

\section{Estadísticos descriptivos de las variables}

La Tabla 2 presenta las medias y las desviaciones estándar de las variables predictoras y de las variables resultantes del estudio. Los resultados de estos análisis descriptivos indicaron que en promedio los adolescentes y jóvenes adultos de la muestra perciben la calidad de la relación con la madre, el padre y su hermano/a como positivas. Con respecto a la evaluación que los participantes hicieron de la calidad de vida y de las dimensiones de competencia social, los resultados mostraron niveles altos de calidad de vida y de empatía y niveles moderados de habilidades para establecer relaciones nuevas.

Adicionalmente, se llevaron a cabo pruebas t para evaluar diferencias entre las variables del estudio de acuerdo con el sexo de los participantes y el sexo del hermano seleccionado. No se encontraron diferencias significativas en las variables del estudio al realizar comparaciones entre hombres y mujeres y de acuerdo con el sexo del hermano

Tabla 2 - Datos demográficos de la muestra de participantes

\begin{tabular}{|c|c|c|}
\hline Variables del estudio & Frecuencia & Porcentaje \\
\hline \multicolumn{3}{|c|}{ Edad participante (años) } \\
\hline $15-18$ & 44 & 42,7 \\
\hline $19-21$ & 42 & 40,78 \\
\hline $22-25$ & 17 & 16,50 \\
\hline \multicolumn{3}{|c|}{ Sexo del hermano (hombres) } \\
\hline Hombre & 28 & 68,3 \\
\hline Mujer & 13 & 31,7 \\
\hline \multicolumn{3}{|c|}{ Sexo del hermano (mujeres) } \\
\hline Hombre & 31 & 50 \\
\hline Mujer & 31 & 50 \\
\hline \multicolumn{3}{|c|}{ Nivel educativo participante } \\
\hline Secundaria & 14 & 13,6 \\
\hline Universidad & 88 & 85,5 \\
\hline Posgrado & 1 & 1 \\
\hline \multicolumn{3}{|l|}{ Nivel socioeconómico } \\
\hline Bajo & 27 & 26,2 \\
\hline Medio & 63 & 61,1 \\
\hline Alto & 12 & 11,7 \\
\hline
\end{tabular}




\section{Edad del padre}

39-49

50-59

60-69

\section{Edad de la madre}

34-44

45-54

55-61

\section{Nivel educativo padre}

Secundaria

Universidad

Postgrado

\section{Nivel educativo madre}

Secundaria

Universidad

Postgrado

\section{Estado civil padres}

Casados

Unión libre
49

40

12

33

32,4

54

15

32

49

48,5

20

19,8

41

41

49

10

10

85

83,3

17

16,7

Asociaciones entre las variables centrales del estudio

Con el fin de evaluar las asociaciones que existen entre la percepción de los adolescentes y jóvenes de su relación con los padres y hermanos, la calidad de vida y la competencia social se llevaron a cabo análisis de correlación. Los resultados confirmaron parcialmente las hipótesis con relación a las asociaciones entre las variables del estudio. En general, se encontró que la calidad de las relaciones familiares se asoció significativamente con las variables resultantes del estudio. Sin embargo, análisis particulares de las correlaciones mostraron asociaciones diferenciales entre las variables para los hombres y para las mujeres participantes en el estudio (ver Tabla 3).

Tabla 3 - Análisis de correlación de las variables del estudio para hombres y mujeres

\begin{tabular}{lcccccc}
\hline Variables del estudio & 1 & 2 & 3 & 4 & 5 & 6 \\
\hline 1.Calidad relación papá & - &, 18 &, $16^{* *}$ &, $51^{* *}$ &, 20 &,- 09 \\
2.Calidad relación mamá &, 19 & - &, $40^{* *}$ &, $41^{* *}$ &, $40^{* *}$ &, 12 \\
3.Calidad relación hermano &, 45 &, 24 & - &, 18 &, $44^{* *}$ &, $34^{*}$ \\
4.Calidad de vida &, $51^{* *}$ &, $30^{*}$ &, $54^{* *}$ & - &, $66^{* *}$ &, 25 \\
5.Habilidad para establecer relaciones &, $34^{* *}$ &, 05 &, $45^{* *}$ &, $57^{* *}$ & - &, 30 \\
6.Empatía &, 16 &, 04 &, $26^{*}$ &, $57^{* *}$ &, $50^{* *}$ & - \\
\hline
\end{tabular}

Nota. Hombres $n=41$, mujeres $n=62$. Las correlaciones de los hombres se presentan arriba de la diagonal y las de las mujeres debajo de la diagonal.

$* * \mathrm{p}<, 01 * \mathrm{p}<, 05$ 
Con respecto a las mujeres, la percepción de la calidad de las relaciones familiares (con papá, mamá, y hermano/a) se asoció significativamente con el nivel de calidad de vida percibido por los participantes ( $r=, 51, \mathrm{r}=, 30$ y r=,54 respectivamente), mientras que en el caso de los hombres la calidad de la relación con los padres (no con el hermano/a) se asoció significativamente con el nivel de calidad de vida $(r=, 51$ y $r=, 41)$. Los resultados relacionados con las dimensiones de competencia social resaltan la importancia de la relación con el padre del sexo opuesto. En el caso de las mujeres, se encontraron asociaciones significativas entre la calidad de la relación con el padre y la habilidad para establecer relaciones $(r=, 34)$; mientras que para los hombres la habilidad para establecer relaciones se asoció con la calidad de la relación con la madre $(r=, 40)$. La calidad de las relaciones con hermanos mostró asociaciones significativas tanto en las mujeres como en los hombres tanto con la habilidad para establecer relaciones ( $r=, 45$ y $r=, 44$ respectivamente) como con la empatía ( $r=, 26$ y $r=, 34$ respectivamente).

\section{Análisis de Regresión}

Se llevaron a cabo análisis de regresión con el fin de evaluar las preguntas sobre los efectos de las variables predictoras (calidad de la relación con padres y con hermano/a) sobre las variables resultantes (calidad de vida y habilidad para establecer relaciones). Para cada una de las variables se dos modelos de regresión (uno para los hombres y otro para las mujeres). La Tabla 4 presenta una síntesis de los modelos evaluados

Tabla 4 - Análisis de regresiones con procedimiento simultáneo para calidad de vida y la habilidad para establecer relaciones.

\begin{tabular}{lccc}
\hline \multicolumn{1}{c}{ Modelo } & & \\
\hline \multicolumn{1}{c}{ Calidad de vida } & Beta & $p$ \\
\hline $\begin{array}{l}\text { Hombres }(n=41) \\
\text { Calidad relación papá }\end{array}$ & $F(2,38)=10,88 p=, 00$ &, 45 &, 00 \\
Calidad relación mamá &, 33 &, 02 \\
$R^{2}=, 36 \quad R^{2}$ Ajustado= ,33 & $F(3,57)=13,16 p=, 00$ & & \\
Mujeres ( $n=62)$ & &, 32 &, 01 \\
Calidad relación papá &, 16 &, 13 \\
Calidad relación mamá &, 36 &, 00 \\
Calidad relación hermano & & \\
$R^{2}=, 41 \quad R^{2}$ Ajustado= 38 & & & \\
\hline
\end{tabular}

Habilidad para establecer relaciones

\begin{tabular}{lll}
\hline $\begin{array}{l}\text { Hombres }(n=41) \\
\text { Calidad relación mamá }\end{array}$ & $F(2,38)=6,48 \quad p=, 00$ &, 08 \\
$\begin{array}{l}\text { Calidad relación hermano } \\
R^{2}=, 25 \quad \mathrm{R}^{2} \text { Ajustado }=, 21\end{array}$ &, 33 &, 04 \\
$\begin{array}{l}\text { Mujeres }(n=62) \\
\text { Calidad relación papá }\end{array}$ & & \\
Calidad relación hermano & & \\
$R^{2}=, 23 \quad R^{2}$ Ajustado=,20 & $, 38)=8,85 p=, 00$ &, 00 \\
\hline
\end{tabular}


Calidad de vida. El modelo de calidad de vida para la muestra de hombres fue significativo ( $F$ $(2,38)=10,88 p=, 00)$. Este modelo explicó el $36 \%$ de la varianza (33 \% ajustado) y la calidad de la relación con la mamá, así como la calidad de la relación con el papá tuvieron un efecto significativo sobre la calidad de vida de los participantes. En el caso de las mujeres, el modelo fue igualmente significativo $(F(3,57)=13,16 p=, 00)$; este modelo incluyó tanto la calidad de la relación con madre y padre como la calidad de la relación con el hermano y explicó el $41 \%$ de la varianza (38 \% ajustado). De las tres variables predictoras solamente la calidad de la relación con el papá y con el/la hermano/a tuvieron un efecto significativo sobre la calidad de vida de las participantes.

Habilidad para establecer relaciones. El modelo de la habilidad para establecer relaciones para la muestra de hombres fue significativo $(F(2,38)=6,48 p=, 00)$. Este modelo explicó el $25 \%$ de la varianza ( $21 \%$ ajustado) y en este caso las variables predictoras fueron la calidad de la relación con la mamá y la calidad de la relación con el/la hermano/a. Solamente la calidad de la relación con el hermano tuvo un efecto significativo sobre la habilidad de los hombres para establecer relaciones. En el caso de las mujeres, el modelo fue igualmente significativo $(F(2,59)=8,85 p=, 00)$; este modelo incluyó la calidad de la relación con el papá y la calidad de la relación con el/la hermano/a como variables predictoras y explicó el $23 \%$ de la varianza (20 \% ajustado). Solamente la calidad de la relación con el/la hermano/a tuvo un efecto significativo sobre la habilidad de las mujeres para establecer relaciones.

\section{Discusión}

El propósito central de esta investigación fue evaluar la asociación entre la calidad de la relación con padres y hermanos y la calidad de vida y competencia social de adolescentes y jóvenes adultos colombianos. A continuación se presenta una breve discusión de los resultados, las limitaciones del estudio y recomendaciones para investigaciones en el futuro sobre este tema.

\section{Relaciones familiares y calidad de vida}

En términos generales, los resultados obtenidos a partir del análisis de correlaciones y el análisis de regresión corroboraron las hipótesis planteadas en relación con la asociación entre la calidad de la relación con el padre, la madre, y el/la hermano/a escogido y la calidad de vida de los adolescentes y jóvenes. Sin embargo, se encontraron diferencias en los patrones de asociación de estas variables, así como en su efecto sobre la calidad de vida dependiendo del sexo de los participantes. De igual manera, los patrones de asociación entre la calidad de las relaciones en diferentes sistemas (padrehijo, madre-hijo, hermanos) y la habilidad para establecer relaciones sociales variaron de acuerdo con el sexo del participante.

Congruente con los planteamientos de Lauren \& Mooney (2008), estos resultados permiten resaltar dos aspectos que han sido objeto de estudio en investigaciones sobre los efectos de las relaciones familiares sobre los adolescentes y jóvenes. Por una parte, los efectos específicos que pueden tener las relaciones con diferentes miembros de la familia sobre el bienestar, desarrollo y calidad de vida de los adolescentes y, por otro lado, la manera como la importancia de las relaciones con diferentes miembros de la familia en la adolescencia y la juventud puede variar de acuerdo con el sexo del individuo. En relación con los efectos específicos de diferentes miembros de la familia, estudios sobre los efectos diferenciales del estilo de crianza materno y paterno sobre el bienestar de los jóvenes han demostrado que tanto el padre como la madre contribuyen de manera independiente a la felicidad de los hijos (Cheng \& Furham, 2004). De manera similar, otros estudios han mostrado que no sólo los jóvenes evalúan de manera diferente su relación con el padre y la madre, sino que la calidad de cada una de estas relaciones incide de manera diferente en variables como la calidad de vida y el bienestar psicológico de los jóvenes (Orbuch et al., 2005) y en algunas dimensiones de la calidad de la relación entre hermanos (Ripoll, Carrillo y Castro, 2009).

Con respecto a las diferencias asociadas al sexo en los efectos de las relaciones con los padres sobre el bienestar psicológico de adultos jóvenes, no existen resultados definitivos que permitan 
concluir de qué manera se asocian estas variables en hombres y mujeres jóvenes. Diferentes estudios han encontrado patrones de asociación particulares, que parecerían variar de acuerdo con la cultura. Por ejemplo, estudios en países como India (Parmar \& Rohner, 2005) y Estados Unidos (Rohner, 1998) han encontrado que el ajuste psicológico de hombres jóvenes se encuentra más fuertemente asociado con la calidad de la relación con el padre, que a la calidad de la relación con la madre. Sin embargo, otros estudios realizados en Colombia y Puerto Rico han encontrado que al controlar estadísticamente el efecto de la relación con la madre, la asociación entre calidad de la relación con el padre y el ajuste psicológico de hombres jóvenes deja de ser significativa (Ripoll-Núñez \& Alvarez, 2008). Así mismo, en países como Korea los estudios han indicado que el ajuste psicológico de las mujeres, y no de los hombres, tiene una asociación más fuerte con la calidad de la relación tanto con el padre, como con la madre (Chyung \& Lee, 2008).

A pesar de que se han planteado diferentes hipótesis para explicar estos resultados acerca de la influencia de patrones de crianza y socialización en diferentes culturas, no existe un consenso al respecto. Es necesario entonces continuar investigando los patrones de asociación y los efectos específicos que tienen las relaciones con el padre y la madre sobre indicadores de calidad de vida y ajuste psicológico de jóvenes y adolescentes. Así mismo, las investigaciones citadas anteriormente y el presente estudio hacen evidente la necesidad de evaluar la asociación entre estas variables para hombres y mujeres por separado.

De otro lado, esta investigación encontró que no sólo los efectos de la calidad de la relación con cada uno de los padres, sino también los efectos de la relación entre hermanos sobre la calidad de vida varían de acuerdo con el sexo de los jóvenes. Así, mientras en las mujeres la calidez en la relación entre hermanos y la relación con el padre se encontraban asociados significativamente con su calidad de vida; en el caso de los hombres la calidez en la relación entre hermanos no tuvo un efecto significativo independiente cuando se controló estadísticamente el efecto de la calidad de la relación con cada uno de los padres. Estos resultados ponen en evidencia la necesidad de considerar las relaciones entre hermanos, cuando se evalúan los diferentes subsistemas familiares que influyen en la calidad de vida de adolescentes y jóvenes. A pesar de que en la literatura se reconoce la importancia de la relación afectiva entre hermanos en el desarrollo de los adolescentes, son pocos los estudios en los que se evalúan simultáneamente los efectos particulares de la relación con cada uno de los padres y la relación entre hermanos en la calidad de vida de adolescentes y jóvenes. Por lo tanto, como lo señalan otros especialistas en psicología del desarrollo (Sanchez-Queija \& Oliva, 2003) se requieren más estudios como el presentado en este manuscrito, que permitan establecer los efectos diferenciales de diversos sistemas de relación en la calidad de vida de mujeres y hombres en esta etapa del desarrollo.

\section{Relaciones familiares y competencia social}

En relación con los efectos de diferentes sistemas de relación sobre las competencias sociales de jóvenes y adolescentes, los resultados de este estudio revelaron que tanto en hombres como en mujeres la calidad de la relación entre hermanos posee un efecto único y significativo sobre la habilidad para establecer relaciones sociales con pares. Este efecto de la calidad de la relación entre hermanos fue estadísticamente significativo, aún cuando se controló la asociación entre las competencias sociales y la calidad de las relaciones con el padre y la madre. Como lo han indicado otras investigaciones, los efectos positivos de la relación entre hermanos sobre el desarrollo de competencias sociales pueden explicarse desde diferentes puntos de vista. Por ejemplo, desde los postulados de la teoría de la mente, las conversaciones e intercambios exitosos en las relaciones entre hermanos se asocian con el desarrollo de habilidades socio-cognitivas en los niños, como entender y demostrar empatía ante las emociones de los demás (Cutting \& Dunn, 2006). Así mismo, las oportunidades que proveen las relaciones entre hermanos de relacionarse con alguien con una manera de pensar distinta a la propia influyen positivamente en el establecimiento y mantenimiento de relaciones sociales con pares tanto en la niñez como en la adolescencia (Cassidy, Fineberg, Brown, \& Perkins, 2005). Por otra parte, es posible plantear que en el caso particular de adolescentes y jóvenes, las relaciones con hermanos influyen más fuertemente en el desarrollo de sus competencias sociales, porque lo/as her- 
mano/as comparten características generacionales (por ejemplo, experiencias, intereses, visión del mundo) con los pares con quienes se relacionan estos individuos en diferentes contextos sociales. Así, a diferencia de la relación con los padres, la relación con los hermanos provee oportunidades para practicar habilidades específicas que son útiles en el intercambio social con pares.

\section{Limitaciones del estudio y futuras investigaciones}

A pesar de las contribuciones señaladas anteriormente, los resultados de esta investigación sólo pueden considerarse como preliminares. Las características de la muestra de participantes y del método de obtención de la muestra hacen difícil generalizar los resultados de esta investigación a una población específica. Se requiere entonces replicar esta investigación empleando una muestra probabilística, que represente adecuadamente las características de los adolescentes y jóvenes colombianos.

Por otra parte, los resultados presentados en esta investigación no nos permiten concluir sobre relaciones de tipo causal entre las variables estudiadas. El diseño transversal de este estudio, sólo nos permite concluir sobre la existencia de relaciones significativas entre la calidad de los diferentes sistemas de relación, la calidad de vida y las competencias sociales de los jóvenes. Sin embargo, se requiere de estudios de tipo longitudinal para esclarecer la naturaleza de las relaciones entre estas variables.

Finalmente, esta investigación se basó únicamente en el reporte de los jóvenes y adolescentes para evaluar la asociación entre variables estudiadas. En estudios posteriores será necesario obtener reportes de otros miembros de la familia y de pares tanto sobre las competencias sociales de los jóvenes, como sobre la calidad de las relaciones afectivas en la familia. El uso de múltiples informantes y diferentes métodos de obtención de información (cuestionarios de auto reporte, observaciones) reducirá los errores causados por el uso de métodos comunes de evaluación.

\section{Referencias}

Armsden, G. C., \& Greenberg, M. T. (1987). The inventory of parent and peer attachment: Individual differences and their relationship to psychological well-being in adolescence. Journal of Youth Adolescence, 16, 427-453.

Berlin, L. J., Cassidy, J., \& Appleyard, K.( 2008). The influence of early attachments on other relationships. En J. Cassidy \& Ph. Shaver (Eds.), Handbook of Attachment (pp. 333-365) (2ºd). New York: Guilford.

Blake, J., Richardson, B., \& Bhattacharya, J. (1991). Number of siblings and sociability. Journal of Marriage and Family, 53, 271-283.

Botello-Harbaum, M., Nansel, T., Haynie, D. L., Iannotti, R. J., \& Simons-Morton, B. (2008). Responsive parenting is associated with improved type 1 diabetes-related quality of lifeChild: Care, Health and Development, 34, 675-681.

Cassidy, K., Fineberg, D., Brown, K., \& Perkins, A. (2005) Theory of Mind May Be Contagious, but You Don’t Catch It From Your Twin. Child Development, 76 (1), 97 -106.

Cheng, H., \& Furnham, A. (2004). Perceived parental rearing style, self-esteem and self-criticism as predictors of happiness. Journal of Happiness Studies, 5, 1-21.

Chyung, Y., \& Lee, J. (2008). Intimate partner acceptance, remembered parental acceptance in childhood, and psychological adjustment among Korean college students in ongoing intimate relationships. Cross-Cultural Research, 42, 77-86.

Cutting, A., \& Dunn, J. (2006) Conversations with siblings and with friends: Links between relationship quality and social understanding. British Journal of Developmental Psychology, 24, 73-87. 
Diener, E., Suh, E. M., Lucas, R. E., \& Smith, H. L. (1999). Subjective well-being: Three decades of progress. Psychological Bulletin, 125, 276- 302.

Diwan, R. (2000). Relational wealth and the quality of life. Journal of Socio-Economics, 29, 305340.

Dunn, J. (2000). State of the art: Siblings. The Psychologist, 13, 244- 248.

Edwards, L., \& López, S. (2006). Perceived family support, acculturation, and life satisfaction in Mexican American youth: A mixed methods exploration. Journal of Counseling Psychology, 53, 279- 287.

Furnham, A. \& Cheng, H. (2000). Perceived parental behavior, self-esteem and happiness. Social Psychiatry and Psychiatric Epidemiology, 35, 463-470.

Gringeri, A., \& Von Mackensen, S. (2008). Quality of life in haemophilia. Haemophilia, 14, 1925.

Kenny, M. E., \& Rice, K. G. (1995). Attachment to parents and adjustment in late adolescent college students: Current status, applications, and future considerations. The Counseling Psychologist, 23, 433- 456.

Ladd, G. (2005). Children's peer relations and social competence: A century of progress. New Haven: Yale University Press.

Lamarche, V., Brendgen M., Vitaro, F., Pérusse, D., \& Dionne, G. (2006). Do friendship and sibling relationships provide protection against peer victimization in a similar way? Social Development, 15, 373-393.

Laursen, B., \& Mooney, K. S. (2008). Relationship network quality: Adolescent adjustment and perceptions of relationships with parents and friends. American Journal of Orthopsychiatry, 78, 47-53

Lockwood, R., Kitzmann, K., \& Cohen, R. (2001). The impact of sibling warmth and conflict on children's social competence with peers. Child Study Journal, 31, 47-70.

McCoy, K., Brody, G., \& Stoneman, Z. (2002). Temperament and the quality of best friendships: effect of same-sex sibling relationships. Family Relations, 51, 248-255.

Moss, E., St.Laurent, D., Dubois- Comtois, K., \& Cyr,Ch. (2005). Quality of attachment at school age: Relations between child attachment behavior, psychosocial functioning, and school performance. En K. Kerns, \& R. Richardson (Eds,), Attachment in middle school (pp. 189-211). New York: The Guilford Press.

Musitu, G., \& Cava, M. J. (2002). El rol del apoyo social en el ajuste de los adolescentes. Intervención Psicosocial, 12, 179-192.

Noller, P. (2005). Sibling relationships in adolescence: Learning and growing together. Personal relationships, 12, 1- 22.

Oliva, A., \& Arranz, E. (2005). Sibling relationships during adolescence. European Journal of Developmental Psychology, 2, 253-270.

Orbuch, T. L., Parry, C., Chesler, M., Fritz, J., \& Repetto, P. (2005). Parent-child relationships and quality of life: resilience among childhood cancer survivors. Family Relations, 54, 171-183.

Parke, R. (2004). Development in the Family. Annual Review of Psychology, 55, 365-399.

Parmar, P., \& Rohner, R. P. (2005). Relations among perceived intimate partner acceptance, remembered parental acceptance, and psychological adjustment among young adults in India. Ethos, 33, 402-413. 
Power, M., Bullinger, M., Harper, A., \& The World Health Organization Quality of Life Group. (1999). The world health organization WHOQOL-100: tests of the universality of quality of life in 15 different cultural groups worldwide. Health Psychology, 18, 495-505.

Power, M. J. (2003). Quality of life. En S. J. López \& C. R. Snyder. (Eds). Positive Psychological Assessment: A Handbook of Models and Measure (pp. 427-441). Washington: APA.

Rice, K., \& Cummins, P. N. (1996). Late adolescence and parent perceptions of attachment: An exploratory study of personal and social well-being. Journal of Counseling and Development, 75, 50-57.

Ripoll-Núñez, K., \& Alvarez, C. (2008). Perceived intimate partner acceptance, remembered parental acceptance and psychological adjustment among Colombian and Puerto Rican youths and adults. Cross-Cultural Research, 42, 23-34.

Ripoll, K., Carrillo, S., \& Castro, J. (2009). Relación entre hermanos y ajuste psicológico: Los efectos de la calidad de la relación padres-hijos. Avances en Psicología Latinoamericana, 27 (1), 122-139.

Rohner, R. P. (1998). Father love and child development. Current Directions in Psychological Science, 7, 157-161.

Rohner, R. P. (2005). Parental Acceptance-Rejection Questionnaire (PARQ): Test Manual. En R. P. Rohner, \& A. Khaleque (Eds.), Handbook for the study of parental acceptance and rejection, 4th Edition, (pp.43-106). Storrs, CT: Rohner Research Publications.

Sánchez -Queija, I., \& Oliva, A. (2003). Vínculos de apego con los padres y relaciones con los iguales durante la adolescencia. Revista de Psicología Social, 18 (1), 71-86.

Skevington, S. M. (2002). Advancing cross-cultural research on quality of life: observations drawn from the WHOQOL development. Quality of Life Research, 11, 135-144.

Thrinke, S. J., \& Bartholomew, K. (1997). Hierarchies of attachment relationships in young adulthood. Journal of Social and Personal Relationships, 39, 77- 91.

WHOQOL Group. (1998). The world health organization quality of life assessment (WHOQOL): development and general psychometric properties. Social Science and Medicine, 46, 15691585.

Zimmermann, J. J., Eisemann, M. R., \& Fleck, M. P. (2008). Is parental rearing an associated factor of quality of life in adulthood? Quality of Life Research, 17, 249-255.

Vera, E., Thakral, Ch., González, R., Morgan, M., Conner, W., Caskey, E., Bauer, A., Mattera, L., Clark, S., Bena, K., \& Dick, L. (2008 ). Subjective well-being in urban adolescents of color. Cultural diversity and ethnic minority psychology, 14, 224-233. 